\title{
Detailed Modelling of Thermal Units From a Price-Taker's Perspective
}

\author{
Marijn Maenhoudt \\ Dept. Electrical Engineering (ESAT), Div. ELECTA \\ Katholieke Universiteit Leuven (KU Leuven) \\ Leuven, Belgium \\ marijn.maenhoudt@esat.kuleuven.be
}

\author{
Geert Deconinck \\ Dept. Electrical Engineering (ESAT), Div. ELECTA \\ Katholieke Universiteit Leuven (KU Leuven) \\ Leuven, Belgium \\ geert.deconinck@esat.kuleuven.be
}

\begin{abstract}
Generation Companies (GenCos) acting as pricetakers, schedule their plants according to market price forecasts. As these GenCos do not consider active market price setting as a strategy to increase profits, their performance solely relies on (1) price forecast accuracy and (2) the representation of a plant's operational characteristics. This paper strives at constituting a reference work for thermal plant modelling from a price-taker's perspective. Two main contributions can be distinguished. Firstly it summarizes relevant contributions from literature and merges them into one single, complete formal mixed integer linear problem (MILP) model design. Secondly it adds new features to advance the state-of-the-art and to enhance the applicability of the model in practice. The document is finalised by assessing the model performance by applying it to an exemplary case study.
\end{abstract}

Keywords - thermal unit commitment, modelling, price-based, electricity market, mixed integer linear problem, price-taker

\section{INTRODUCTION}

In a liberalized electricity market GenCos need to deploy the available capacity of generation plants in their portfolio mix to anticipate unknown demand. Perfect demand anticipation is impossible as demand is not exactly known on beforehand and needs to be forecasted. The forecast uncertainty approaches zero close to energy delivery time. Perfect demand anticipation is thus only achievable if a GenCo can adjust its portfolio energy output continuously according to updated forecasts. However, the technologies out of which a GenCo's portfolio consists are not endlessly controllable and/or flexible.

Three types of plants can be distinguished in a typical GenCo's portfolio mix: renewable energy sources, energy storage sources and thermal energy sources. Renewable energy sources are very flexible as significant differences in energy output level can occur between two subsequent moments in time, but they only allow for very limited control since - even with the installation of power electronics allowing for curtailment - the instantaneous energy output is dictated by weather conditions, which are stochastic by nature. Today's most economically relevant energy storage sources are hydroelectric energy sources. This technology is both flexible and controllable. Its exploitation however is highly dependent on geographical requirements such as the presence of a river and a high elevation, thereby drastically reducing its applicability. Thermal plants are highly relied on to cover a large share of the total demand as this technology is highly controllable. Each thermal plant however is subject to fixed inter-temporal constraints, allowing for a thermal flexibility spectrum ranging from low flexibility - generally the case for thermal plants with low variable costs - to a reasonably high flexibility - generally for thermal plants with high variable costs. This inverse relationship causes inflexible plants to cover base-load demand while more flexible plants are used to cover peak-load demand.

Demand uncertainty can only be met by supply flexibility in absence of widely available or economically viable energy storage technologies. Increasing supply flexibility is achieved by either developing better forecast tools or by modelling the dynamic technical characteristics of all plants in the GenCo's portfolio as accurately as possible. Comprehensive models allow for better tuning between the different generation technologies by maximizing each plant's utilization efficiency.

The focus of this paper lies on modelling thermal plants for in 2009 these technologies attributed to $81.9 \%$ of the total EU electricity production [1]. Much literature has focused on modelling thermal units, however a single reference work combining all progress in this area seems to be missing in existing literature. The contribution of this paper lies in organising relevant existing literature, in combining it into one single comprehensive modelling framework and in advancing the level of detail of such models. The model's performance is empirically assessed on exemplary cases.

The content of the paper is organised as follows. In section I the motivation for creating the paper is presented. Before discussing the evolution in thermal plant modelling approaches and assessing the pros and cons of selected approaches more in detail in Section III, the most important economic and technical constraints to be taken into account when designing such approach must be introduced in Section II. Section IV builds further on the previous sections by presenting the formal model design of a portfolio consisting solely of thermal plants. An exemplary case study in section $\mathrm{V}$ empirically examines the performance of the proposed model. Finally the document is concluded by recapitulating the main objectives of this paper.

\section{THERMAL PLANT CHARACTERISTICS}

The workings of a thermal plant are subject to many technical limitations. Pang et al. concisely elaborate a range of technical constraints when formulating a cost-based thermal unit commitment [2]. In general these dynamic constraints are inherent to the units out of which the plant is composed and can be divided in three categories.

Some research leading to these results has received funding from the European Community's Seventh Framework Programme under grant agreement FP7-239456-optimate 


\section{A. Economic Constraints}

Variable costs are dependent on the fuel price, unit loading and thermal efficiency. As fuel input increases, the marginal increase in power output decreases due to increased heat leakage with increasing operating temperature [3]. The slope of the curve is influenced by the fuel price and thermal efficiency, the latter varying in value in terms of unit loading. The variable cost is thus best approximated by a strictly increasing quadratic function in terms of unit loading. The power output level corresponding to the unit's maximum efficiency working point is denoted as the rated power of the unit. If the GenCo resides in an area with an emission trading system in place then the associated cost can be integrated in the fuel cost.

Start-up costs are incurred when a thermal unit undergoes a cold start and needs to ramp up until the rated minimum power output is reached. Start-up costs are dependent on the temperature of the unit which cools down exponentially after a shut-down event. Start-up costs increase thus exponentially in terms of unit down time. The unit's maintenance costs in contrast increase due to additional component stress when a unit is not allowed to fully cool down after shutting down.

Shut-down costs are associated to the ramping down of a thermal unit from the rated minimum power down to the point the unit actually stops operating. Generally shut-down costs are independent of the time the unit has been operating but not respecting the unit's minimum up time would lead to extra component stress and thus increased the maintenance costs.

\section{B. Operating Constraints}

Thermal plants are subject to strict technical operating requirements which greatly reduce their flexibility. Since a plant is composed out of multiple units - each having their own flexibility constraints - the flexibility of the total plant is not constant in time. The majority of flexibility constraints can be perceived within two main categories: power output level (in)flexibility and time (in)flexibility.

The minimum and maximum power levels define the stable power output region within which a unit can be dispatched. A unit operating within these limits is said to be in production phase. The power limits of all individual units define the power limits and production phase of the whole plant. As each individual unit can be forced to go off-line due to maintenance or unexpected component failure, the limits of the plant can be considered dynamic. Additionally, as starting up individual units requires labour by engineers, potential crew constraints limit the number of units to be started up simultaneously. The thermal unit is not immediately operational at the minimum power level after start-up. The unit first experiences a start-up phase where it gradually increases its power level from zero up to the minimum power. Similarly, the shut-down phase of the unit imposes a gradual decrease in power output from the rated minimum power down to zero. Lastly the unit can also operate in a hot stand-by phase: the unit is not producing any power but its core is sustained at high temperature levels. A thermal unit typically engages in its hot stand-by phase when the cost of a future cold start-up exceeds the cost of the fuel needed to generate enough heat and the costs for a warm start-up.
A dispatched thermal unit is strictly subject to upward and downward gradient (or ramping) limits. Gradient limits are dependent on the operational phase the unit resides. The gradient limit is independent of the actual power output level within one operational phase, but the maximum upward gradient limit might differ from the maximum downward gradient limit in the same operational phase.

Technically the thermal unit can strictly increase or decrease its power output at variable ramping rates. Reversing the ramping direction however is not straightforward. The unit first must maintain a stable power output for a minimum time duration before reversing the ramping direction in order to relieve component stresses. A final explicit timing constraint relates to the engineering consideration or manufacturing specification that a unit normally operates for a minimum time duration before it is shut down. Similarly a minimum unit down time is imposed between successive operational cycles. Additional stress on technical components - and thus additional costs - has to be taken into account if minimum up and down times are not respected.

\section{Reserve Requirements}

Energy markets can be categorized in terms of the time horizon between the organisation of the market and the real time energy delivery. Forward energy markets have a time horizon of a day or longer, while any market with a shorter time horizon is classified under reserve markets. This categorisation has emerged historically as thermal plants do not offer large flexibility after day-ahead gate closure time due to the above-mentioned technical limitations. Renewable energy sources on the other hand benefit from more accurate forecasts the closer to delivery time.

The GenCo thus needs to take uncertain reserve market liquidity into account as well as the provision of ancillary services to the Transmission System Operator (TSO). These services consist of providing spinning and non-spinning reserves, reactive power, automatic generation control, etc.

Finding the optimal trade-off between profit, forecast error risk, thermal flexibility risk and system security risk has been the subject of many studies [4], [5], [6], [7]. The majority of literature approaches the problem by means of stochastic modelling. In this paper stochastic multimarket optimisation is omitted but the possibility that stochastic optimisation can be integrated in the present framework by means of for example Monte Carlo simulation is explicitly stated.

\section{SHORT-TERM THERMAL UNIT COMMITMENT MODELS}

Modelling thermal units holds a historical track record. Pang et al. approached the problem from a cost minimization approach. The heuristic truncated dynamic programming method was used to minimize production and start-up costs, while fulfilling spinning reserve, load, and crew requirements. Technical constraint were limited to minimum and maximum power limits. The start-up and shut-down phase of the plant is not explicitly modelled but approximated by a step function. The method only guarantees near optimal feasible schedules due to the enormous computational efforts needed by the procedure [2]. 
Merlin et al. resort to Lagrangian relaxation for solving cost-based thermal unit commitment problems. The problem includes load and spinning reserve requirements, minimum and maximum power limits, and the minimum up and down times. The solution method aims at minimizing production and startup costs [8]. Lagrangian relaxation is used in [9] where the problem is formulated more in detail by imposing ramping limits and exponential start-up costs but omitting shut-down costs in the problem formulation.

Special focus on optimizing the scheduling of spinning reserves has been considered in [6]. The aim of the method is to reduce the problem of over-commitment of reserves in Lagrangian relaxation based unit commitments. Chung-Li Tseng et al. use a mixed-integer linear problem formulation to optimally schedule reserve requirements by considering spinning reserve requirements as a soft constraint [7].

Arroyo et al. pioneered in thermal unit commitment model design by formulating the problem from the perspective of the GenCo and not the one of the system operator by formulating the problem as a price-based unit commitment [10]. The profitmaximising objective function includes expected wholesale and reserve market prices, production costs, non-linear start-up costs and shut-down costs. Plant production and gradient limits are considered as well as minimum up and down times. The impact on the level of detail is assessed by means of an exemplary case study. In [11], the start-up and shut-down trajectories were formulated in detail. Finally, by focusing on computational efficiency, the number of constraints and their complexity has been reduced in [12]. However, start-up and shut-down costs are considered constant in time and the new formulation does not smoothly model the gradient limits transition between the operational phases. The plant is considered as a single unit instead of a composition of multiple individual units.

Niknam et al. use Benders Decomposition to solve a costbased thermal unit commitment problem [13]. The problem formulation takes into account the plant power limits, gradient limits and the minimum up and down times. The main focus of this research work is to investigate model convergence and execution time by analysing several test cases rather than advancing the state-of-the-art of thermal unit commitment problem formulation.

In the next section the proposed thermal price-based unit commitment model is clarified. The proposed model aims at combining all features of the models mentioned in this section. The problem formulation presented here applies to a portfolio composed of thermal plants. Each thermal plant consists of multiple individual units, each with their own operational phases, power limits, gradient limits, costs for starting up and shutting down and minimum up and down times. The model advances the state-of-the-art by providing explicit constraints for modelling start-up and shut-down phases, by expanding the model design to include the hot stand-by phase and by proposing additional equations to ensure a smooth variability in gradient limits when transitioning between the operational phases of a thermal plant. Crew constraints are incorporated to accomplish a robust and self-contained model design compared to the ones discussed earlier.

\section{UNIT COMMITMENT PROBLEM FORMULATION}

The GenCo's objective is to maximise its profits over the time horizon $T$. The profit is calculated as the total revenue $(R)$ generated by the thermal plants minus the total production $(P)$, start-up $(S)$ and shut-down $(D)$ costs. A plant is denoted by index $p$; a unit by index $u$. The portfolio is assumed to consists of $P$ plants, with a single plant containing $U(p)$ units.

$$
\sum_{t}^{T} R(t)-\sum_{t}^{T} P(t)-\sum_{t}^{T} S(t)-\sum_{t}^{T} D(t)
$$

The total energy produced by the GenCo's portfolio mix $Q$ depends on the energy produced by the individual units of which the thermal plants are composed.

$$
\begin{gathered}
Q(t)=\sum_{1}^{P} Q(p, t) \\
Q(p, t)=\sum_{1}^{U(p)} Q(p, u, t)
\end{gathered}
$$

Under the uniform market pricing scheme, the GenCo's revenue per time step equals the amount of energy sold times the hourly expected market price $(P r)$.

$$
R(t)=\operatorname{Pr}(t) * Q(t)
$$

Figure 1 illustrates the four different operational phases of the unit: the start-up phase $(S P)$, shut-down phase $(D P)$, production phase $(P P)$ and hot stand-by phase $(H B)$. Binary values ensure the correct transition from one phase to the other. The start-up moment $(S M)$ initiates the start-up phase, while the shut-down moment $(D M)$ terminates the shut-down phase. A similar definition holds for the hot stand-by "start" (HSM) and "end" moment $(H D M)$. Lastly, when the unit is producing power - including the power produced during start-up or shutdown phases - the unit is considered operational $(O N)$ in contrast to when no power is produced such as during the hot stand-by phase or after the shut-down phase.

$$
\begin{gathered}
S M(p, u, t)+H B(p, u, t)-H S M(p, u, t) \leq 1 \\
D M(p, u, t)+H B(p, u, t)+H D M(p, u, t) \leq 1 \\
S M(p, u, t-1)+D M(p, u, t) \leq 1 \\
S M(p, u, t-1)-D M(p, u, t)+H D M(p, u, t-1) \\
-H S M(p, u, t)=O N(p, u, t)-O N(p, u, t-1)(8) \\
H S M(p, u,-1 t)-H D M(p, u, t)=H B(p, u, t) \\
\quad-H B(p, u, t-1) \leq 1(9) \\
S P(p, u, t)+D P(p, u, t)+P P(p, u, t)=O N(p, u, t) \\
S P(p, u, t-1) \leq 1-D P(p, u, t) \\
D P(p, u, t-1) \leq 1-S P(p, u, t) \\
S M(p, u, t-1) \leq S P(p, u, t) \\
D M(p, u, t) \leq D P(p, u, t-1)
\end{gathered}
$$




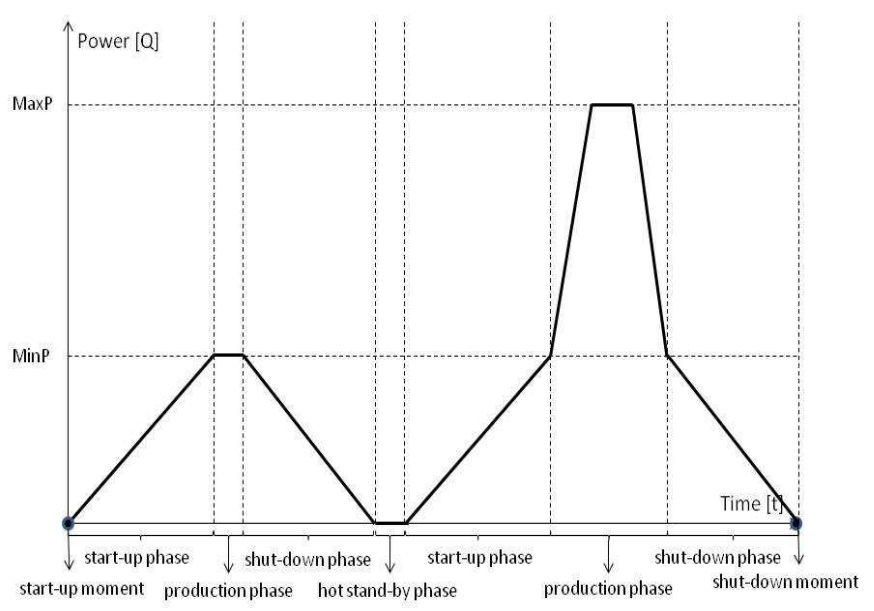

Fig. 1. Unit's Operational Phases

As indicated in section II the maximum gradient limits are dependent on the unit's operational phases. The maximum start-up (MaxStUp), shut-down (MaxShDown) and the rated upward (MaxUp) and downward (MaxDown) gradient limits can be defined for the start-up, shut-down and production phase respectively. These ramping speeds are expressed in accordance to the granularity of the problem formulation $(t)$. In turn the minimum $(\operatorname{Min} P)$ and maximum $(\operatorname{Max} P)$ power limits link the gradient limits of the unit with its power output level.

$$
\begin{array}{r}
Q(p, u, t)-\operatorname{MinP}(p, u) * O N(p, u, t) \\
\geq-\operatorname{Min} P(p, u) *(S P(p, u, t)+D P(p, u, t) \\
Q(p, u, t)-\operatorname{MinP}(p, u) * O N(p, u, t) \\
\leq(\operatorname{Max} P(p, u)-\operatorname{Min} P(p, u)) * P P(p, u, t) \\
Q(p, u, t) \leq Q(p, u, t-1)+\operatorname{Max} U p(p, u, t-1) \\
Q(p, u, t) \geq Q(p, u, t-1)-\operatorname{MaxDown}(p, u, t-1)
\end{array}
$$

Additional equations must be included in the problem formulation to ensure a smooth valuation for the gradient limits between the various phases. The transition from start-up phase to production phase occurs when the unit's output during startup becomes higher than the minimum power limit minus the maximum start-up gradient. Analogously, the transition from production phase to shut-down phase occurs when the power output becomes lower than the minimum power limit plus the maximum downward gradient. Calculating the duration until the power output crosses the minimum power threshold given the transition ranges defined above leads to the correct gradient up and down limits.

The formal constraint formulation requires the power output to be divided into operational and transition phases. Figure 2 illustrates the principle graphically and illustrates the classification of the power output in five ranges $(r)$. A binary value $H$ ensures the hierarchical order between these ranges: the unit can only provide power output (Out) to a higher range if the power outputs of all previous ranges have been supplied.

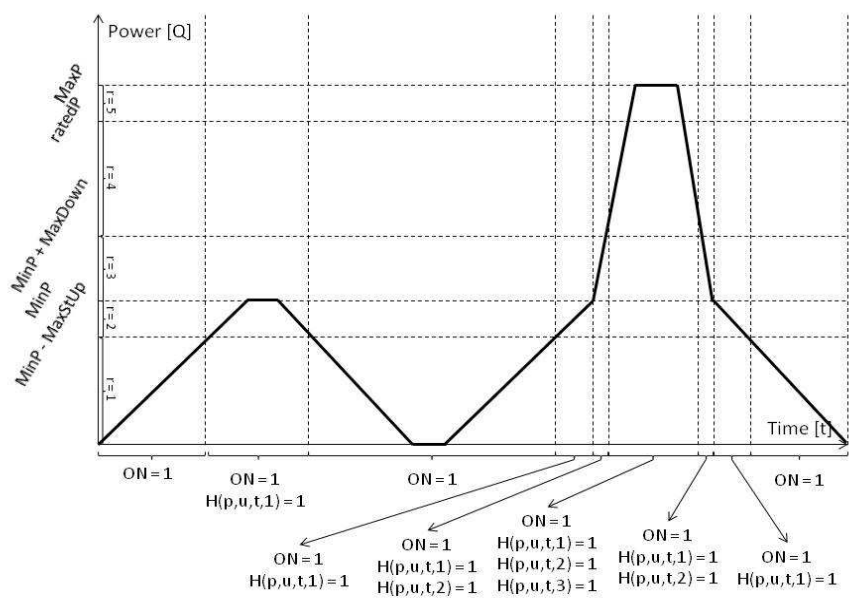

Fig. 2. Unit's Gradient Modelling

$$
\begin{array}{rl}
(\operatorname{MinP}(p, u)-\operatorname{MaxStUp}(p, u)) * & H(p, u, t, r=1) \\
\leq \operatorname{Out}(p, u, t, r=1)
\end{array}
$$

$\operatorname{Out}(p, u, t, r=1)$

$$
\leq(\operatorname{MinP}(p, u)-\operatorname{MaxStUp}(p, u)) * O N(p, u, t)
$$

$\operatorname{MaxStUp}(p, u) * H(p, u, t, r=2) \leq \operatorname{Out}(p, u, t, r=2)$

$\operatorname{Out}(p, u, t, r=2) \leq \operatorname{MaxStUp}(p, u) * \operatorname{Out}(p, u, t, r=1)(22)$

$\operatorname{MaxDown}(p, u) * H(p, u, t, r=3) \leq \operatorname{Out}(p, u, t, r=3)(23)$

$\operatorname{Out}(p, u, t, r=3) \leq \operatorname{MaxDown}(p, u) * \operatorname{Out}(p, u, t, r=2)(24)$

$(\operatorname{rated} P(p, u)-\operatorname{Min} P(p, u)-\operatorname{MaxDown}(p, u))$

$* H(p, u, t, r=4) \leq \operatorname{Out}(p, u, t, r=4)$

$\operatorname{Out}(p, u, t, r=4) \leq(\operatorname{rated} P(p, u)-\operatorname{MinP}(p, u)-$ $\operatorname{Max} \operatorname{Down}(p, u)) * \operatorname{Out}(p, u, t, r=3)$

$$
\begin{array}{r}
(\operatorname{Max} P(p, u)-\operatorname{rated} P(p, u)) * H(p, u, t, r=5) \\
\leq \operatorname{Out}(p, u, t, r=5)
\end{array}
$$

$\operatorname{Out}(p, u, t, r=5) \leq(\operatorname{Max} P(p, u)-\operatorname{rated} P(p, u))$

$$
\text { * Out }(p, u, t, r=4)
$$

A linear interpolation obtains the correct maximum upward and downward gradient limits. Finally, a constraint ensuring power production consistency needs to be added.

$$
\begin{gathered}
\operatorname{MaxUp}(p, u, t) \leq \operatorname{Max} \operatorname{Mp}(p, u) \\
+(\operatorname{MaxStUp}(p, u)-\operatorname{Max} \operatorname{dp}(p, u)) * \\
(\operatorname{MaxStUp}(p, u)-\operatorname{Out}(p, u, t, r=2)) / \operatorname{MaxStUp}(p, u)
\end{gathered}
$$

$$
\operatorname{MaxDown}(p, u, t) \leq
$$$$
\operatorname{Out}(p, u, t, r=3)+\operatorname{Max} \operatorname{ShD} o(p, u)-
$$

$(\operatorname{MaxShDo}(p, u) * \operatorname{Out}(p, u, t, r=3) / \operatorname{MaxDown}(p, u))(30)$

$$
Q(p, u, t)=\sum_{1}^{5} \operatorname{Out}(p, u, t, r)
$$


The minimum stable power output level duration for reversing the ramping direction from upward to downward (MinDuraUp) and from downward to upward (MinDuraDown) are imposed by two binary variables. The first one detects an increase in unit power output (Inc) while the other detects power output decrements $(D e c)$. The formulation assumes no reverse ramping can occur during either the start-up phase or the shut-down phase and does not allow constraint relaxation in exchange of higher incurred costs. Obviously, a unit cannot both increase and decrease its power output at the same time.

$$
\operatorname{Max} U p(p, u, t) \leq \operatorname{Max} U p(p, u) * \operatorname{Inc}(p, u, t)
$$

$\operatorname{MaxDown}(p, u, t) \leq \operatorname{MaxDown}(p, u) * \operatorname{Dec}(p, u, t)(33)$ $\min \operatorname{DuraUp}(p, u) * \operatorname{Inc}(p, u, t)+$ $\sum_{t^{\prime}=t-\operatorname{MinDuraU}}^{t}(p, u)+1 \operatorname{Dec}\left(p, u, t^{\prime}\right) \leq \operatorname{MinDuraUp}(p, u)(34)$

$$
\begin{aligned}
& \operatorname{minDuraDown}(p, u) * \operatorname{Dec}(p, u, t)+ \\
& \sum_{t^{\prime}=t-\operatorname{MinDuraDown}(p, u)+1}^{t} \operatorname{Inc}\left(p, u, t^{\prime}\right) \leq
\end{aligned}
$$

$\operatorname{MinDuraDown}(p, u)$

$$
\begin{gathered}
S P(p, u, t)+S M(p, u, t) \leq \operatorname{Inc}(p, u, t) \\
D P(p, u, t)+\operatorname{DM}(p, u, t) \leq \operatorname{Dec}(p, u, t) \\
\operatorname{Inc}(p, u, t)+\operatorname{Dec}(p, u, t) \leq 1
\end{gathered}
$$

$$
\begin{aligned}
& \operatorname{Inc}(p, u, t) \leq S P(p, u, t)+P P(p, u, t)+S M(p, u, t) \\
& \operatorname{Dec}(p, u, t) \leq D P(p, u, t)+P P(p, u, t)+D M(p, u, t)
\end{aligned}
$$

The crew constraint can be indirectly approximated by defining a maximum amount of simultaneous unit start-ups (MaxUnits) that can be handled by the scheduled workforce in a single plant. Literature shows no evidence of a similar constraint for shutting down a thermal unit. The formulation is omitted but it is noted that such constraint can easily be added thanks to the design of the proposed model.

$$
\sum_{1}^{U(p)} S M(p, u, t) \leq \operatorname{MaxUnits}(p)
$$

The quadratic production cost - or fuel cost - of a single unit is approximated by linear interpolation between the previously defined ranges $r$ by defining the marginal increase in costs per power output unit (Slope). This representation allows incorporating the maximum cost-efficiency unit working point at the rated power $($ ratedP). Figure 3 illustrates the linear approximation of the quadratic variable cost curve. Note that the intercept of the curve denotes fuel consumption during the hot stand-by phase.

$$
P(p, u, t)=\sum_{1}^{R}(\operatorname{Out}(p, u, t, r) * \operatorname{Slope}(p, u, r))+
$$
CostAtZeroPower $(p, u) * O N(p, u, t)+(\operatorname{StByCost}(p, u) *$ $H B(p, u, t)+H S M(p, u, t)+H D M(p, u, t))$

$$
\begin{gathered}
P(p, t)=\sum_{1}^{U(p)} P(p, u, t) \\
P(t)=\sum_{1}^{P} P(p, t)
\end{gathered}
$$

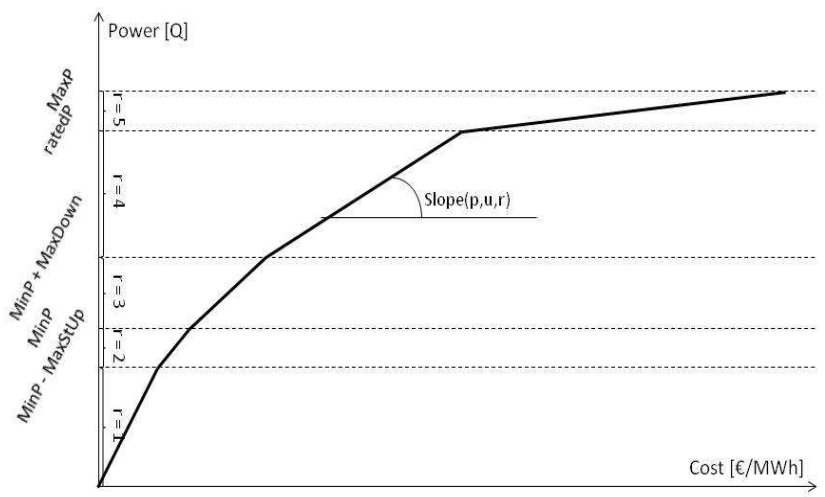

Fig. 3. Unit's Fuel Cost Modelling

Figure 4 illustrates the linear interpolation of the exponential start-up cost curve by the he marginal increase of start-up costs $($ Cost $S)$ in function of the time the unit has been inactive (tDown) in analogy to the definition of the quadratic production cost curve. Note that for illustrative purposes the unit's cooling down time has been arbitrarily chosen at 4 in terms of the problem granularity. Although shut-down costs are assumed independent of the unit's active operation time, the explicit formulation is presented here to account for timedependent maintenance costs for violating minimum up times.

$$
\begin{aligned}
& S(p, u, t) \geq \operatorname{CostS}(p, u, t D o w n) \\
& *\left(\operatorname{SM}(p, u, t)-\sum_{t^{\prime}=t-t \text { Down }}^{t-1} O N\left(p, u, t^{\prime}\right)\right) \\
& S(p, u, t) \geq 0 \\
& S(p, t)=\sum_{1}^{U(p)} S(p, u, t) \\
& S(t)=\sum_{1}^{P} S(p, t) \\
& \text { * }\left(D M(p, u, t)-\sum_{t-t U p}^{t-1}\left(1-O N\left(p, u, t^{\prime}\right)\right.\right. \\
& D(p, u, t) \geq 0 \\
& D(p, t)=\sum_{1}^{U(p)} D(p, u, t) \\
& D(t)=\sum_{1}^{P} D(p, t)
\end{aligned}
$$

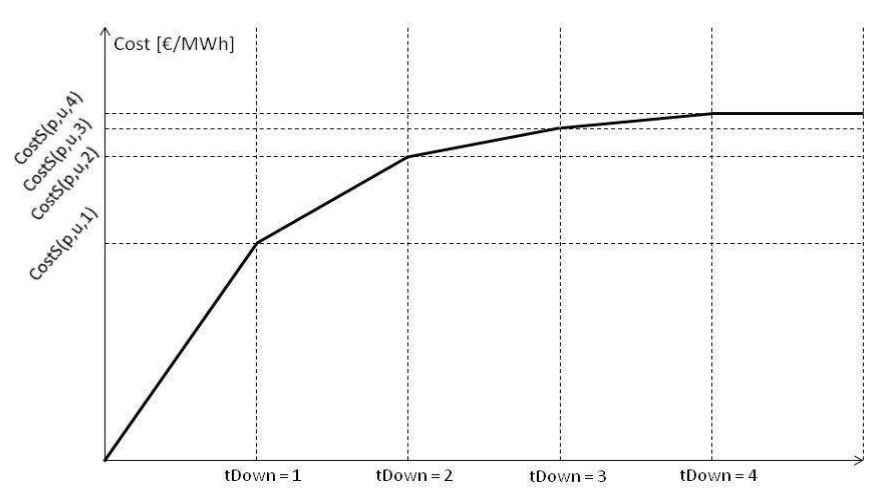

Fig. 4. Unit's Start-Up Cost Modelling 


\section{CASE Study}

Two studies are conducted to evaluate the performance of the proposed methodology. The first study focuses on the novel modelling of smooth ramping transition between operational phases. A similar assessment approach as in [11] is conducted with its own model as benchmark case. The second study is conducted for illustrative purposes in order to gain preliminary insights of model performance under uncertainty.

\section{A. Detailed Ramping Modelling}

The case study involves a single thermal plant consisting of a single unit. The production cost is $\$ 38 / \mathrm{MWh}$ with a minimum variable cost of $\$ 100 / \mathrm{h}$. Shut-down costs are $\$ 56$ and the cost for starting up is $\$ 850$. Based on these costs the granularity of the problem is chosen as hourly.

The start-up and shut-down process lasts 3 hours. The average ramping up during the start-up phase is $38 \mathrm{MW} / \mathrm{h}$. The average ramping down rate during shut-down is $40 \mathrm{MW} / \mathrm{h}$.

The capacity of the unit is 294 MW. Minimum up and down times are 3 hours. The ramping up and down limits are 60 and $50 \mathrm{MW} / \mathrm{h}$ respectively. The unit has already been operating for 11 hours and produces $170 \mathrm{MW}$ during the last hour of the previous day.

Table I shows the price forecasts, the optimal output calculated by [11] and the optimal power output calculated by the method from this paper. The latter solution has been calculated using a $2.27 \mathrm{GHz}$ processor and $4 \mathrm{~GB}$ of RAM using CPLEX 12 under GAMS 23.7.2 [14].

TABLE I. PRICE DATA AND OPTIMAL GENERATION SCHEDUle

\begin{tabular}{|c|c|c|c|}
\hline Hour & $\begin{array}{c}\text { Market Price } \\
\text { [\$/MWh] }\end{array}$ & $\begin{array}{c}\text { Power Output } 1 \\
{[\mathrm{MW}]}\end{array}$ & $\begin{array}{c}\text { Power Output } 2 \\
{[\mathrm{MW}]}\end{array}$ \\
\hline 1 & 26,950 & 120 & 120 \\
\hline 2 & 25,817 & 112 & 78,4 \\
\hline 3 & 22,209 & 70 & 38,4 \\
\hline 4 & 21,219 & 30 & 0 \\
\hline 5 & 19,668 & 0 & 0 \\
\hline 6 & 19,800 & 0 & 0 \\
\hline 7 & 24,167 & 0 & 38 \\
\hline 8 & 26,950 & 37 & 76 \\
\hline 9 & 40,700 & 75 & 115.2 \\
\hline 10 & 44,110 & 112 & 175.2 \\
\hline 11 & 45,177 & 172 & 235.2 \\
\hline 12 & 47,025 & 232 & 294 \\
\hline 13 & 47,773 & 292 & 294 \\
\hline 14 & 46,200 & 294 & 294 \\
\hline 15 & 44,033 & 294 & 294 \\
\hline 16 & 44,550 & 294 & 294 \\
\hline 17 & 46,200 & 294 & 294 \\
\hline 18 & 48,950 & 294 & 294 \\
\hline
\end{tabular}

\begin{tabular}{c|c|c|c}
\hline Hour & $\begin{array}{c}\text { Market Price } \\
\text { [\$/MWh] }\end{array}$ & $\begin{array}{c}\text { Power Output 1 } \\
\text { [MW] }\end{array}$ & $\begin{array}{c}\text { Power Output 2 } \\
\text { [MW] }\end{array}$ \\
\hline 19 & 48,840 & 294 & 294 \\
\hline 20 & 45,100 & 294 & 294 \\
\hline 21 & 43,252 & 294 & 294 \\
\hline 22 & 44,000 & 244 & 244 \\
\hline 23 & 44,000 & 194 & 194 \\
\hline 24 & 25,850 & 144 & 144 \\
\hline
\end{tabular}

The faster ramping is achieved since it only takes the plant 9.6 minutes to complete the transition from $120 \mathrm{MW}$ to 112 MW in hour 1. While the benchmark method does not take into account 50 minutes of ramping down in shut-down phase, the proposed method in this paper does so. The plant not only ramps down faster in hours 1 to 5 where the price is too low to produce, it also starts producing earlier without infringing upon its minimum down time constraints.

The case study illustrates that a smoother transition between the different phases allows a more detailed production output solution. This addition of constraints modelling the smooth transition between phases results in a profit of $\$ 19385$ compared to a profit of $\$ 16773.5$ as reported in [11], equivalent to a profit increase of $15.6 \%$. Assuming $\$ 100 / \mathrm{h}$ hot stand-by costs, the profit increases to $\$ 20264$ since expensive start-up costs are avoided, or equivalent to an increase of $20.8 \%$.

\section{B. Illustrative Case}

Assume a GenCo owning a plant composed of three units with reference data as defined in table II. The data in the tables is based upon data found in [15].

TABLE II. DATA SHEET ILlustrative CASE

\begin{tabular}{c|c|c|c}
\hline Parameter & Base & Middle & Peak \\
\hline MaxP & 400 & 300 & 250 \\
\hline RatedP & 380 & 285 & 238 \\
\hline MinP & 100 & 100 & 75 \\
\hline MaxStUp & 30 & 40 & 50 \\
\hline MaxShDown & 30 & 40 & 50 \\
\hline MaxUp & 100 & 80 & 88 \\
\hline MaxDown & 100 & 80 & 88 \\
\hline MinDuraUp & 8 & 5 & 2 \\
\hline MinDuraDown & 8 & 5 & 2 \\
\hline
\end{tabular}

\begin{tabular}{c|c|c|c}
\hline Fuel Cost & Base & Middle & Peak \\
\hline 0 & 0 & 0 & 0 \\
\hline MinP & 1000 & 3500 & 3750 \\
\hline RatedP & 3800 & 9975 & 11900 \\
\hline MaxP & 4000 & 10500 & 12500 \\
\hline StandBy & 150 & 120 & 75
\end{tabular}




\begin{tabular}{c|c|c|c|c|c|c|c}
\hline \multicolumn{4}{|c|}{ Start Up Cost } & \multicolumn{4}{c}{ Shut Down Cost } \\
\hline hour & Base & Middle & Peak & hour & Base & Middle & Peak \\
\hline 1 & 100 & 80 & 50 & 1 & 100 & 80 & 50 \\
\hline 2 & 500 & 400 & 250 & 2 & 500 & 400 & 250 \\
\hline 3 & 800 & 650 & 400 & 3 & 500 & 400 & 250 \\
\hline 4 & 1000 & 800 & 500 & 4 & 500 & 400 & 250 \\
\hline
\end{tabular}

The purpose of this example is to illustrate the impact of different levels of modelling accuracy on method performance. In order not to be limited to a single specific case, a Monte Carlo simulation is performed under Gaussian price uncertainty with hourly mean prices chosen as the values presented in table I and an hourly standard deviation of $10 \%$. Each scenario is run 100 times. Initially, each unit is assumed not to be operating.

The impact of crew constraints, explicit modelling of startup and shut-down phases instead of assuming an instantaneous start-up or shut-down to/from the minimum power level output, and the impact of a non-linear fuel cost representation are represented in table III. Note that the non-linear fuel cost representation is made under the assumption that there is an increase in marginal fuel cost when producing at a higher level than the rated power output level. The relative increase is denoted between brackets.

TABLE III. Illustrative CASE: Results

\begin{tabular}{c|c|c|c}
\hline $\begin{array}{c}\text { Maximum } \\
\text { simultaneous } \\
\text { start-ups }\end{array}$ & $\begin{array}{c}\text { Explicit } \\
\text { modelling of } \\
\text { phases? }\end{array}$ & $\begin{array}{c}\text { Fuel } \\
\text { cost } \\
\text { representation }\end{array}$ & $\begin{array}{c}\text { Expected } \\
\text { profit [\$] }\end{array}$ \\
\hline 3 & Yes & Linear & 279151 \\
\hline 3 & No & Linear & 289522 \\
\hline 3 & Yes & Non-linear (10\%) & 277992 \\
\hline 2 & Yes & Linear & 278786 \\
\hline 1 & Yes & Linear & 278786 \\
\hline
\end{tabular}

The results in table III illustrate that any simplification made when modelling a thermal plant results in a higher expected profit. The general conclusion to be drawn is that the proposed method is more accurate and complete than existing models. Since it is in the best interest of a GenCo not to overestimate expected profits, the existence of such reference model proves to be valuable.

\section{CONCLUSIONS AND FUTURE WORK}

The main motivation for this research work was the observed unavailability of one single comprehensive literature work which concisely collects and presents all existing work related to this research topic and which merges all their findings together in one single formulation. The price-based mixed integer linear unit commitment formulation was chosen in this paper based on its potential to find the optimal solution and its potential to model technical and economic constraints.

The performance of the proposed modelling framework has been illustrated by studying both a comparative and illustrative case. Although a complete performance assessment requires more studies than the ones presented, the value of such comprehensive method has been illustrated: the level of detail is high while the calculation time can be considered negligible.

Still, topics for further research can be pinpointed. The first major topic is the integration of stochastic multimarket risk mitigation as mentioned in II.C. Although multimarket risk mitigation can be integrated as an extension of the current modelling framework, still the formulation needs to be made explicit. A second topic the author is working on is creating a unit commitment formulation from a price-setter perspective, incorporating the self-scheduling effect on emerging market prices. Thirdly this model formulation is restricted to thermal plants only. A contribution in this field of research would lie in the creation of an overview for modelling any type of plant.

\section{REFERENCES}

[1] Eurostat Energy Statistics, DG ECFIN and EEA, "EU Energy in figures and factsheets (revision 2011)," June 2011. [Online]. Available: http://ec.europa.eu/energy/ publications/statistics/statistics_en.htm.

[Accessed October 2011].

[2] C. K. Pang and H. C. Chen, "Optimal Short-Term Thermal Unit Commitment," IEEE Transactions on Power Apparatus and Systems, vol. 95, no. 4, pp. 1336 1346, August, 1976.

[3] D. S. Kirschen and G. Strbac, Fundamentals of Power System Economics, Wiley, 2004.

[4] L. Bo, Z. Ming and L. Gengyin, “An Optimal Approach for Coordinating Scheduling Day-Ahead and Real-Time Energy Market with Risks," in International Conference on Power System Technology (POWERCON), 22-26 October, 2006.

[5] M. Gibescu, E. W. van Zwet, W. L. Kling and R. D. Christie, "Optimal Bidding Strategy for Mixed-Portfolio Producers in a Dual Imbalance Pricing System," in 16th PSCC, Glasgow, Schotland, July 14-18, 2008.

[6] H. B. Gooi, D. P. Mendes, K. R. W. Bell and D. S. Kirschen, "Optimal Scheduling of Spinning Reserves," IEEE Transactions on Power Systems, vol. 14, no. 4, pp. 1485 - 1492, 1999.

[7] C.-L. Tseng, S. S. Oren, A. J. Svoboda and R. B. Johnson, "Price-Based adaptive spinning reserve requirements in power system scheduling," Electrical Power and Energy Systems, vol. 21, pp. 137 - 145, 1999.

[8] A. Merlin and P. Sandrin, "A New Method for Unit Commitment at Electricite de France," IEEE Transactions on Power Apparatus and Systems, vol. 102, no. 5, pp. 1218 - 1225, 1983.

[9] J. F. Bard, "Short-Term Scheduling of Thermal-Electric Generators using Lagrangian Relaxation," Operation Research, vol. 36, no. 5, pp. 755 - 766, 1988. 
[10] J. M. Arroyo and A. J. Conejo, "Optimal Response of a Thermal Unit to an Electricity Spot Market," IEEE Transactions on Power Systems, vol. 15, no. 3, pp. 1098 - 1104, 2000.

[11] J. M. Arroyo and A. J. Conejo, "Modeling of Start-Up and Shut-Down Power Trajectories of Thermal Units," IEEE Transactions on Power Systems, vol. 19, no. 3, pp. 1562 - 1568, 2004.

[12] M. Carrión and J. M. Arroyo, "A Computationally Efficient Mixed-Integer Linear Formulation for the Thermal Unit Commitment Problem," IEEE Transactions on Power Systems, vol. 21, no. 3, pp. 1371 - 1378, 2006.

[13] T. Niknam, A. Khodaei and F. Fallahi, "A new decomposition approach for the thermal unit commitment problem," Applied Energy 86, pp. 1667 1674, 2009.

[14] "On-line Documentation," GAMS Development Corporation, [Online]. Available: http://www.gams.com/. [Accessed 2011].

[15] E. Delarue, C. De Jonghe, R. Belmans and W. D'haeseleer, "Applying portfolio theory to the electricity sector: Energy versus power," Energy Economics, vol. 33, no. 1, pp. 12 - 23, January, 2011.

\section{BIOGRAPHIES}

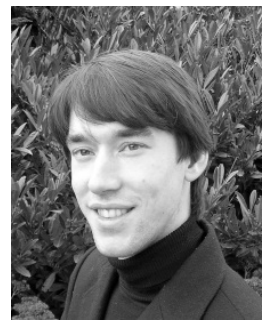

Marijn Maenhoudt (M'09) was born in Belgium in 1984. He studied at the University of Leuven, Leuven, Belgium where he received his B.E and M.E. degree in electrical engineering. He is now enrolled in a $\mathrm{Ph} . \mathrm{D}$. in electrical engineering at the same university with as general topic the strategic behavior exercised by electricity market participants.

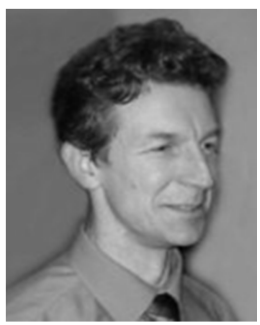

Geert Deconinck (M'88,SM'00) is full professor (gewoon hoogleraar) at the Department of Electrical Engineering at the University of Leuven, Belgium. He performs research on the design and evaluation of architectures for industrial control and smart grids. $\mathrm{He}$ teaches topics related to industrial automation, energy and measurement techniques. He is chairman of the TI society BIRA on industrial automation and a member of the IEEE SMC TC on Infrastructure Systems and Services, a member of the Royal Flemish Engineering Society the Institute of Engineering and Technology (IET), and a senior member of the IEEE (Reliability, Computer and Power \& Energy Societies). 